\title{
Impact of posterior femoral condylar cartilage and posterior intercondylar distance on rotation of femoral component in total knee arthroplasty
}

Teruyuki Miyasaka ${ }^{1,2^{*}}$ (D, Mitsuru Saito ${ }^{2}$, Daisaburo Kurosaka², Ryo Ikeda², Shoki Yamanaka ${ }^{1,2}$ and Keishi Marumo²

\begin{abstract}
Background: Greater accuracy is needed when determining the final femoral component (FC) rotation during total knee arthroplasty (TKA), because this parameter affects soft tissue balance during flexion and patellar tracking. Anatomical markers, such as the epicondylar axis, are typically used to determine the final FC rotation, although intraoperative confirmation may be challenging. Therefore, rotational position is frequently determined with the posterior condylar axis (PCA) as a landmark. However, the thickness of the posterior condylar cartilage has not been considered and may not be represented on preoperative images. We used plain $X$-rays to measure the thickness of the medial and lateral posterior condylar cartilage fragments postoperatively, and investigated the effects of differences in cartilage thickness on final FC rotation.
\end{abstract}

Methods: Fifty knees (19 men, 31 women) underwent primary TKA to treat medial knee osteoarthritis at our hospital between August 2015 and May 2017. All knees were treated using an Attune PS (DePuy Synthes, Inc., Warsaw, IN). We first measured the distance between the posterior femoral condyles, resected the posterior condyle, and measured the thickness of the resected cartilage fragments. We then took X-ray images from a direction tangential to the osteotomy surface, secured the cartilage fragments with digital calipers, and measured the thickness of the cartilage. We investigated the effects of differences in cartilage thickness on final FC rotation of the residual medial and lateral cartilage with a trigonometric function.

Results: Medial condylar cartilage thickness averaged $0.6 \pm 0.5 \mathrm{~mm}$ and the lateral condylar thickness averaged $1.8 \pm 0.6 \mathrm{~mm}$; posterior intercondylar distance averaged $46.1 \pm 3.3 \mathrm{~mm}$ and average impact on rotation of the cartilage remnant was $1.5 \pm 0.9^{\circ}\left(-0.1-3.9^{\circ}\right)$. There may be measurement error of up to $4^{\circ}$ in the maximum values compared with the preoperative plan in cases with short intercondylar distance.

(Continued on next page)

\footnotetext{
* Correspondence: teru5038@gmail.com

'Department of Orthopaedic Surgery, Toshima Hospital, 33-1 Sakaecho, Itabashi-ku, Tokyo 173-0015, Japan

${ }^{2}$ Department of Orthopaedic Surgery, Jikei University School of Medicine,

3-25-8 Nishi-Shimbashi, Minato-ku, Tokyo 105-8461, Japan
}

C C The Author(s). 2020 Open Access This article is licensed under a Creative Commons Attribution 4.0 International License, which permits use, sharing, adaptation, distribution and reproduction in any medium or format, as long as you give appropriate credit to the original author(s) and the source, provide a link to the Creative Commons licence, and indicate if changes were made. The images or other third party material in this article are included in the article's Creative Commons licence, unless indicated otherwise in a credit line to the material. If material is not included in the article's Creative Commons licence and your intended use is not permitted by statutory regulation or exceeds the permitted use, you will need to obtain permission directly from the copyright holder. To view a copy of this licence, visit http://creativecommons.org/licenses/by/4.0/ The Creative Commons Public Domain Dedication waiver (http://creativecommons.org/publicdomain/zero/1.0/) applies to the data made available in this article, unless otherwise stated in a credit line to the data. 
(Continued from previous page)

Conclusions: In cases where the FC external rotation angle is determined using the posterior condyles as landmarks, this angle can be affected by the intercondylar distance, especially in Japanese women who have small physical stature. This angle can potentially be much larger, so caution is advised. Our results suggest that several anatomical landmarks should be referenced to achieve accurate FC rotation.

Keywords: Femoral component, Rotational alignment, Articular cartilage, Total knee arthroplasty, Radiographs, Posterior condyle, Posterior intercondylar distance

\section{Background}

High accuracy is required in performing total knee arthroplasty (TKA) in order to determine the rotational positioning of the femoral component (FC) because it can influence patellar tracking and symmetry of the flexion gap [1-4]. Navigation systems and PatientSpecific Instruments (PSI) have been reported to have relatively good performance, but there have been unfavorable reports with respect to FC rotation [5-9].

The transepicondylar axis or Whiteside's line is often referenced as an anatomical landmark, but verification may be challenging intraoperatively. Therefore, most TKA systems use the posterior condylar axis (PCA) as a landmark intraoperatively for sizing and rotation alignment. However, the cartilage remnant on the posterior condyles varies in thickness medially and laterally and impacts the rotational angle, and is possibly not reflective of preoperative planning estimates. Past reports have described using imaging technology such as computed tomography (CT) arthrograms and magnetic resonance imaging (MRI), and others have documented determining the impacted angle using trigonometry by measuring the cartilage remnant thickness by actually cutting the cartilage remnant on the posterior condyles with a scalpel after making the bone cuts [10-13].

The impact of the posterior femoral condylar cartilage on FC rotation in TKA is defined by the difference in residual cartilage thickness between the posterior femoral condyle and posterior condylar distance. Therefore, we hypothesized that the posterior condylar distance has a greater influence on the rotational position of FC. If this hypothesis is correct, rotational errors of the FC are likely to occur during surgery in patients with small physique.
To clarify this, we obtained plain X-ray images of the cut posterior condyles parallel to the cut surface and measured the cartilage thickness of the intraoperatively excised medial and lateral posterior condyle bonecartilage fragments by utilizing the differences in radiolucency between cartilage and bone. Then, after making the distal femur bone cuts, we measured the distance between the tips of the posterior condyles for each case and used trigonometry to determine the impact on FC rotational position by differences in cartilage remnant thickness.

\section{Methods}

This study was approved by the institutional review board of our institution. From August 2015 to May 2017, a total of 50 knees (19 men, 31 women) at our institution that underwent primary TKA for medial compartment knee osteoarthritis (OA). Average age was $74.9 \pm 7.6$ years, average body mass index was $26.8 \pm 3.8$, and average preoperative hip-knee-ankle angle was $192.2 \pm 6.0^{\circ}$. The characteristics of the patients are summarized in Table 1. A single surgeon performed the surgeries, bone cuts were made using CT-free navigation system (BrainLAB AG, Munich, Germany) for the distal femur and proximal tibia. The Attune ${ }^{\bullet}$ PS knee system (DePuy Synthes, Inc., Warsaw, IN) was used in all cases.

At the start of the operation, a reference frame was attached to the distal femur or the proximal tibia with a bicortical pin. This was followed by surface matching, where the surgeon digitized freely chosen points on the bone surface of both the femur and the tibia. Femoral and tibia cutting blocks were orientated under real-time visualization on the navigation system display. Instruments using standard posterior condyles as landmarks

Table 1 Patient characteristics

\begin{tabular}{llll}
\hline & Total $(n=50)$ & Men $(n=19)$ & Women $(n=31)$ \\
\hline Age (years) & $74.9 \pm 7.6$ & $72.0 \pm 8.8$ & $76.7 \pm 6.3$ \\
Height $(\mathrm{cm})$ & $153.5 \pm 8.7$ & $161.2 \pm 7.4$ & $148.9 \pm 5.7$ \\
Weight $(\mathrm{kg})$ & $63.5 \pm 12.1$ & $71.9 \pm 12.0$ & $58.4 \pm 9.0$ \\
BMl & $26.8 \pm 3.8$ & $27.6 \pm 3.7$ & $26.3 \pm 3.8$ \\
HKA angle (deg) & $192.2 \pm 6.0$ & $193.4 \pm 5.2$ & $191.5 \pm 6.4$ \\
\hline
\end{tabular}

$B M I$ body mass index, HKA angle hip-knee-ankle angle. Values are reported as the mean \pm standard deviation 


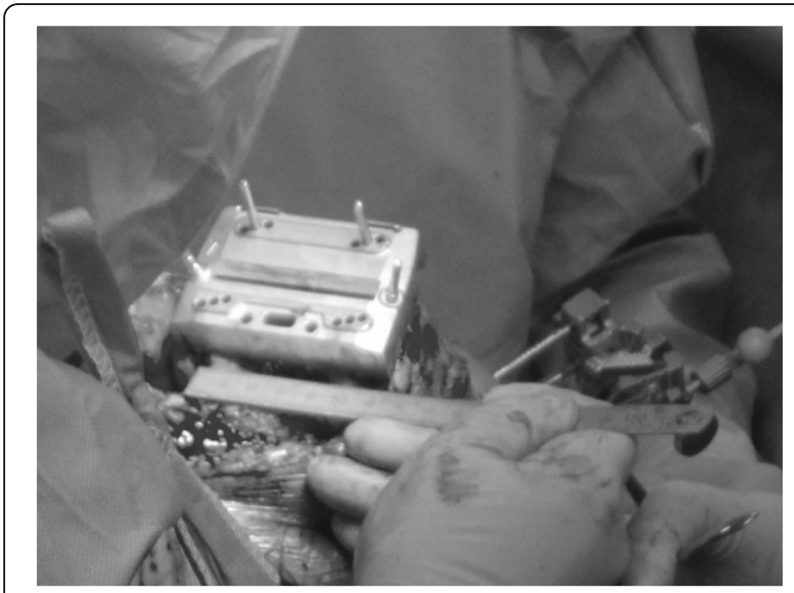

Fig. 1 Measuring posterior femoral intercondylar distance (d)

were used for the posterior condyles. For the proximal tibia resection plane, the resection level was set to $10 \mathrm{~mm}$ from the deepest point of the higher tibia plateau level. Rotational alignment of the tibial tray was achieved by orientating the tray with reference to the medial third of the tibial tuberosity. After resection, all planes were checked using the verification tool of the navigation system. Intraoperatively, after making bone cuts in the distal femur using a CT-free navigation, the posterior femoral intercondylar distance (d) was measured prior to cutting the posterior condyles. The posterior femoral intercondylar distance (d) was defined as the distance between the contact points of the medial and lateral posterior condyles on the rotational alignment jig. The contact points were marked before measuring the distance (Fig. 1). The measurement was taken once during the operation, because if
2 measurements were taken in series, the possibility of the first measurement influencing the second measurement was likely unavoidable. Instead, we (the lead and assistant surgeons) made sure that the ends of the tape measure were accurately placed on the contact points before carefully taking the measurements.

Postoperatively, first a film was placed on a stable table, the surface of which was parallel to the ground. The thickest part of the cartilage fragment was secured with a digital caliper, and the position of the fragment was adjusted by checking it from all directions so that the cut surface was perpendicular to the digital caliper. A stable flat petri dish was placed on the film, and then the main scale of the digital caliper was firmly placed on the petri dish so that the cut surface was stably positioned perpendicular to the film. The cut surface was placed directly under the beam head, and the sample was irradiated perpendicular to the ground. After the Xrays were taken, we confirmed whether the cut surface was in a straight line on the PACS images (Fig. 2). The actual thickness of cartilage on a picture archiving and communication system were calculated, even though the acquired images were enlarged compared with actual objects, because the ratio of thicknesses of bone and cartilage would not change with enlargement (Fig. 3). The impact of differences in cartilage remnant thicknesses on the medial and lateral sides $(1-\mathrm{m})$ on rotational position of FC $(\theta)$ was calculated using trigonometry according to the methods described by Fujii et al. [11] (Fig. 4).

Two observers repeated the measurements twice, and the mean of the four values was regarded as the true value. One observer repeated the measurements 10 times in 3 subjects and assessed the intraobserver

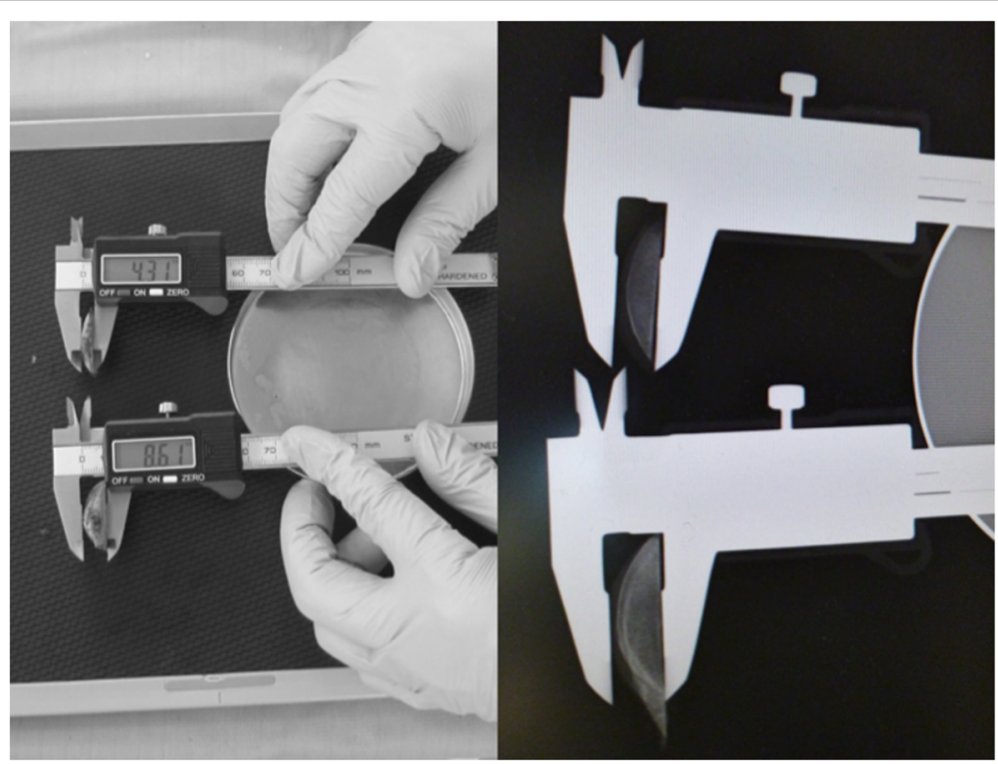

Fig. 2 Radiographs taken parallel to the cut surface 


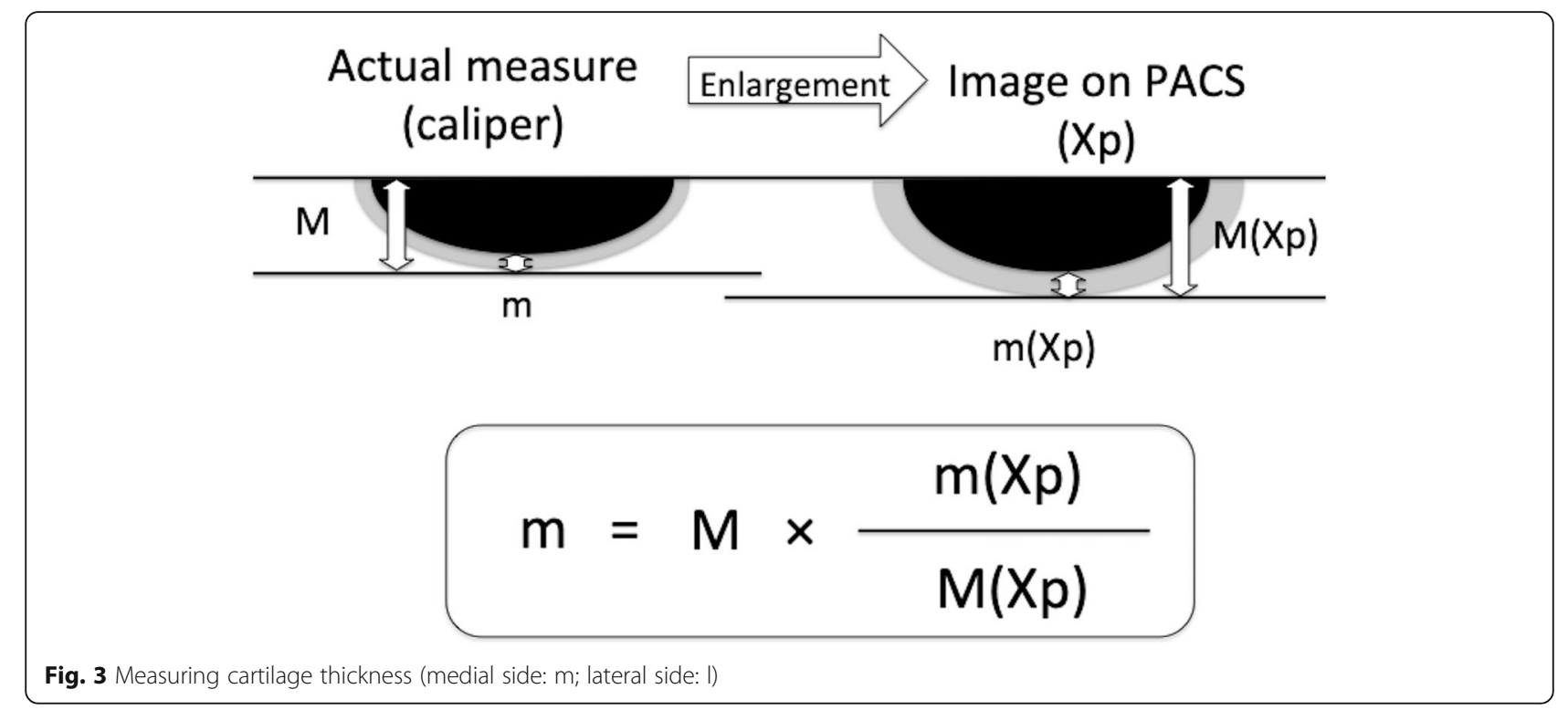

variations of the measurements. The maximum intraobserver difference in the measurements was $0.08 \mathrm{~mm}$ in cartilage thickness (the largest SD was 0.02) and 0.11 $\mathrm{mm}$ in the differences in cartilage remnant thicknesses on the medial and lateral sides (the largest SD was 0.03). Two observers repeated the measurements 10 times in 1 subject and assessed the interobserver variations of the measurements. The maximum interobserver difference was $0.14 \mathrm{~mm}$ in the cartilage thickness (the largest SD was 0.04$)$. In theory, the rotation angle reaches a maximum when the posterior intercondylar distance is at a minimum and the difference between the measurements reaches a maximum. When the posterior intercondylar distance is a minimum of $39 \mathrm{~mm}$, the rotation angle is $0.16^{\circ}$ because the maximum intraobserver difference in the measurements was $0.08 \mathrm{~mm}$ in cartilage thickness. This is $0.21^{\circ}$ because the maximum interobserver difference was $0.14 \mathrm{~mm}$ in terms of cartilage thickness.

Statistical differences between the sexes were assessed using the Mann-Whitney $\mathrm{U}$ test. A $p$-value of $<0.05$ was considered to indicate statistical significance.

\section{Results}

The mean medial condylar cartilage thickness $(\mathrm{m})$ was $0.9 \pm 0.6 \mathrm{~mm}$ in men and $0.4 \pm 0.4 \mathrm{~mm}$ in women; women averaged $0.5 \mathrm{~mm}$ less than men $(p<0.001)$. The mean lateral condylar thickness (l) and differences in cartilage thickness between the medial and lateral condylar cartilages $(1-\mathrm{m})$ averaged $0.2-0.3 \mathrm{~mm}$ less than that in men but was not significantly different between

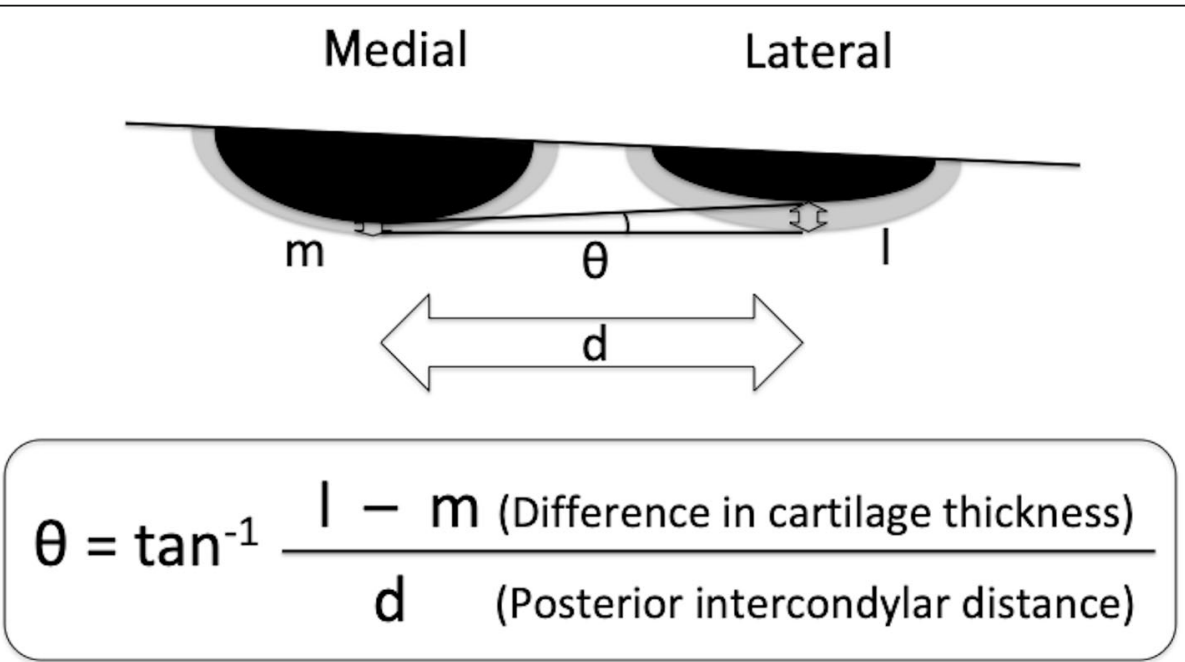

Fig. 4 Calculation method for impact on rotation $(\theta)$ 
Table 2 Comparison of cartilage thickness, posterior intercondylar distance and impact on rotation between men and women

\begin{tabular}{lllll}
\hline & Total $(n=50)$ & Men $(n=19)$ & Women $(n=31)$ & $p$-value Men vs Women \\
\hline Medial side: $\mathrm{m}(\mathrm{mm})$ & $0.6 \pm 0.5(0.0-2.1)$ & $0.9 \pm 0.6(0.3-2.1)$ & $0.4 \pm 0.4(0.0-1.7)$ & $<0.001^{*}$ \\
Lateral side: I $(\mathrm{mm})$ & $1.8 \pm 0.6(0.4-3.0)$ & $1.9 \pm 0.4(0.9-2.5)$ & $1.7 \pm 0.6(0.4-3.0)$ & 0.16 \\
Differences: $\mathrm{I}-\mathrm{m}(\mathrm{mm})$ & $1.2 \pm 0.7(-0.1-2.8)$ & $1.0 \pm 0.6(-0.1-2.0)$ & $1.3 \pm 0.7(0.2-2.8)$ & 0.16 \\
Posterior intercondylar distance: $\mathrm{d}(\mathrm{mm})$ & $46.1 \pm 3.3(39.0-53.0)$ & $49.1 \pm 2.7(44.0-53.0)$ & $44.2 \pm 2.0(39.0-48.0)$ & $<0.001^{*}$ \\
Impact on rotation: $\theta\left(^{\circ}\right)$ & $1.5 \pm 0.9(-0.1-3.9)$ & $1.2 \pm 0.7(-0.1-2.4)$ & $1.7 \pm 0.9(0.3-3.9)$ & 0.12 \\
\hline
\end{tabular}

Data are presented as the mean \pm standard deviation. Medial side, $\mathrm{m}$; lateral side, l; differences, I - m; posterior intercondylar distance, $\mathrm{d}$; impact on rotation, $\theta$. *Significant value

women and men $(p=0.16)$. The mean posterior intercondylar distance (d) was $46.1 \pm 3.3(39.0-53.0) \mathrm{mm}$; the maximum difference was $14 \mathrm{~mm}$. Posterior intercondylar distance averaged $49.1 \pm 2.7(44.0-53.0) \mathrm{mm}$ in men and $44.2 \pm 2.1(39.0-48.0) \mathrm{mm}$ in women, and was about 5 $\mathrm{mm}$ smaller in women than in men $(p<0.001)$. The average impact on rotation of the cartilage remnant was $1.5 \pm 0.9^{\circ}$; the maximum value was $3.9^{\circ}$ with no significant different between women and men $(p=0.12)$ (Tables 2 and 3). Regarding height and posterior intercondylar distance, there was a positive correlation between them both in men and in women, and the correlation was significant in women $(p<0.001)$. Women tended to have smaller intercondylar distance for a given height (Fig. 5). Further, the mean posterior intercondylar distance adjusted for the height was $47.7 \pm 0.4 \mathrm{~mm}$ in men and $45.0 \pm 0.4 \mathrm{~mm}$ in women, indicating a significant difference $(p=0.002$, ANCOVA with height as the covariate). Regarding the impact on rotation and posterior intercondylar distance, Pearson's product-moment correlation analysis confirmed a negative correlation, albeit not significant, in all subjects and in women (Fig. 6). Also, when the rotation angles were compared between the subgroups divided according to the median value, there was a correlation, albeit not significant, in women ( $p=0.06$, Tables 4,5 and 6$)$. Taken together, in women, for subjects with a small intercondylar distance, the impact on rotation of the cartilage remnant tended to be large.

\section{Discussion}

In this study, we measured the intercondylar distance and differences in medial and lateral cartilage thickness for each case to investigate how intercondylar distance and cartilage remnants on the posterior condyles impact rotation. The medial remaining cartilage was significantly thinner in women $(p<0.001)$. Hanna et al. [14] reported that women have increased rates of cartilage loss and progression of cartilage defects at the knee than men. Given that subjects of this study were patients with medial osteoarthritis, the remaining medial cartilage appeared to thin particularly in women. For subjects with small intercondylar distance, the impact on rotation of the cartilage remnant tended to be larger and the greatest discrepancy found was $3.9^{\circ}$ when compared with the value in preoperative planning.

According to reports from various perspectives $[10-$ $13]$, the impact of cartilage remnants on rotation averages $1.1-1.7^{\circ}$, which is in agreement with the average of $1.5^{\circ}$ from the present study (Table 1 ). Asada et al. [10] studied CT arthrograms of 31 knees and reported that the influence of posterior condyle cartilage on rotation was $1.1^{\circ}$ on average and $2.1^{\circ}$ at greatest. Tashiro et al. [13] reported this effect using MRI. However, the articular cartilage thickness shown on CT or MRI could vary depending on imaging resolution. Furthermore, in these studies, osteotomy might not have been performed according to preoperative three-dimensional planning or the actual influence on rotation might have not been

Table 3 Results from the present and previous studies

\begin{tabular}{|c|c|c|c|c|c|}
\hline Author/ Journal & Methodology & $\begin{array}{l}\text { Medial side: } \mathrm{m} \\
(\mathrm{mm})\end{array}$ & $\begin{array}{l}\text { Lateral side: I } \\
(\mathrm{mm})\end{array}$ & $\begin{array}{l}\text { Posterior intercondylar distance: } \\
d(\mathrm{~mm})\end{array}$ & $\begin{array}{l}\text { Impact on rotation: } \\
\theta\left(^{\circ}\right)\end{array}$ \\
\hline $\begin{array}{l}\text { Asada et al. The Knee } \\
\text { (2012) }\end{array}$ & CT Arthrography & & & & $1.1 \pm 0.7(-0.3-2.1)$ \\
\hline Tashiro et al. KSSTA (2012) & MRI & & & & $1.7 \pm 0.71 .5 \pm 0.7$ \\
\hline $\begin{array}{l}\text { Fujii et al. Surg Radiol Anat } \\
\text { (2012) }\end{array}$ & $\begin{array}{l}\text { Cartilage excision by } \\
\text { scalpel }\end{array}$ & $0.7 \pm 0.7(0.0-3.3)$ & $2.1 \pm 0.7(0.0-3.8)$ & & $1.7 \pm 1.3(0.0-4.6)$ \\
\hline $\begin{array}{l}\text { Hamada et al. J Med Invest } \\
\text { (2017) }\end{array}$ & $\begin{array}{l}\text { MPR imaging and } \\
\text { navigation }\end{array}$ & & & & $1.7 \pm 1.2$ \\
\hline Present study & Radiograph & $0.6 \pm 0.5(0.0-2.1)$ & $1.8 \pm 0.6(0.4-3.0)$ & $46.1 \pm 3.3(39.0-53.0)$ & $1.5 \pm 0.9(-0.1-3.9)$ \\
\hline
\end{tabular}

CT computed tomography, MPR multiplanar reformation, MRI magnetic resonance imaging; Data are presented as the mean \pm standard deviation 


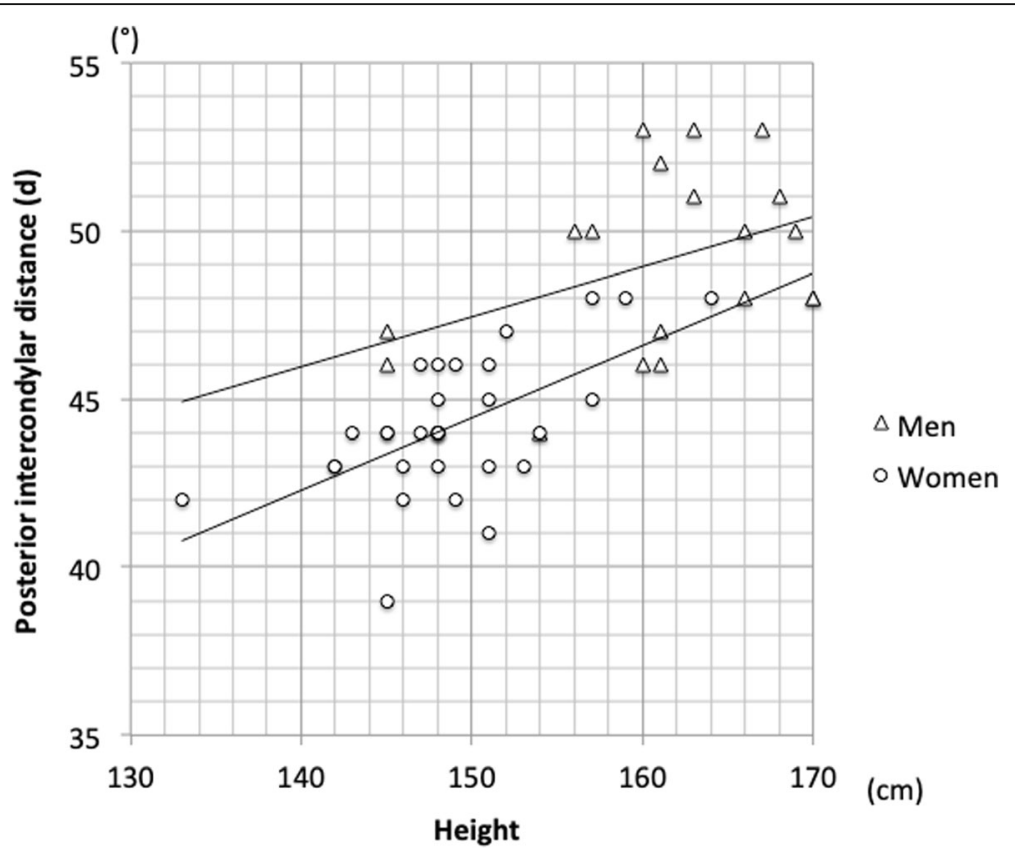

Fig. 5 Association between height and posterior intercondylar distance Men: $r=0.404, p=0.086$; women: $r=0.601, p<0.001$ (Pearson's correlation coefficient)

assessed correctly because the removed osteochondral fragments were not measured directly. Fujii et al. [11] gave no details in their reports of intercondylar distances but did excise cartilage remnants with a scalpel from posterior condyles that were actually cut. They evaluated the differences in thickness before and after excision as the thickness of the cartilage remnant and reported the average as $1.7^{\circ}$ and $4.6^{\circ}$ at maximum. They also reported the average of the medial condylar cartilage as $0.7 \pm 0.7$ $(0.0-3.3) \mathrm{mm}$ and of the lateral condylar cartilage as $2.1 \pm 0.7(0.0-3.8) \mathrm{mm}$. Cartilage from our study was smaller, with an average of $0.61 \pm 0.54(0.00-2.05) \mathrm{mm}$ on the medial condyle and $1.78 \pm 0.56(0.39-2.99) \mathrm{mm}$ on the lateral condyle; the greatest value for the medial

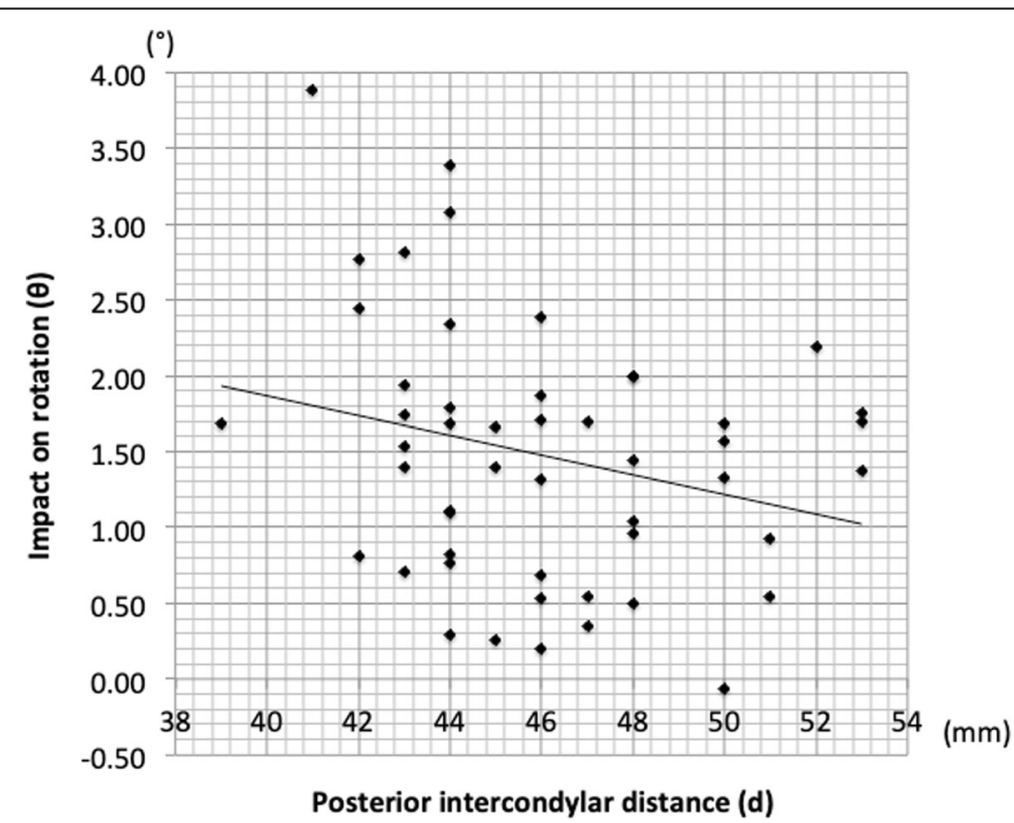

Fig. 6 Association between posterior intercondylar distance $(d)$ and impact on rotation $(\theta)$. All subjects: $r=-0.219, p=0.126 ;$ men: $r=0.267, p=$ 0.269; women: $r=-0.299, p<0.103$ (Pearson's correlation coefficient) 
Table 4 Comparison of external rotation angle in all subjects by subgroups divided according to median value

\begin{tabular}{|c|c|c|c|c|c|}
\hline & & stance (subgro & he $\mathrm{m}$ & & $p$-value \\
\hline & $\overline{\text { Sho }}$ & $46 \mathrm{~mm})$ & Lor & $6 \mathrm{~mm})$ & \\
\hline & $n$ & Data & $\mathrm{n}$ & Data & \\
\hline Angle & 24 & $1.647 \pm 0.949$ & 26 & $1.258 \pm 0.720$ & 0.113 \\
\hline
\end{tabular}

Data are presented as the mean \pm standard deviation. $p$-value: Welch's t-test

side was $1.25 \mathrm{~mm}$ and for the lateral side was $0.81 \mathrm{~mm}$. This may be due to the accuracy of excising the cartilage with a scalpel or from the process of repositioning the bone-cartilage fragment into a caliper after the cartilage had already been measured with a caliper and was then scraped off. The measuring technique we used is performed by taking a single plain X-ray image of the excised bone fragments, and thus is relatively easy, low cost, and has no radiation exposure.

Varying the distal femoral cuts would impact the posterior condyle cuts. Therefore, for the distal femoral cuts, we used a navigation system that is considered to have high accuracy [5-7]. Not only is the affected angle of rotation due to cartilage remnant on posterior condyles influenced by differences in medial and lateral cartilage thickness, but as demonstrated by trigonometric calculations, it is also affected by the intercondylar distance. However, there are no detailed reports on these issues. In the present study, among subjects with small intercondylar distance, the greatest discrepancy was almost $4^{\circ}$ compared with the value obtained during preoperative planning. In addition, 8 subjects who had a discrepancy of $>2.4^{\circ}$ also had a posterior intercondylar distance averaging $43.3 \pm 1.6(41-46) \mathrm{cm}$ and all were below the average posterior intercondylar distance of $46.1 \mathrm{~cm}$.

Ishimaru et al. [15] reported that Japanese women had a relatively narrower femoral width for a given AP length compared with men. Also, our results reveal that posterior intercondylar distance tended to be smaller in women than in men for a given height. Moreover, there was no significant difference in the cartilage thickness between men and women (Differences: $1-m$ ). Thus, in patients with medial compartment OA, if preoperative planning is performed using images where information on cartilage cannot be obtained, or in cases where the external rotation angle of the $\mathrm{FC}$ is determined using the posterior condyles as landmarks, this angle can be affected by the intercondylar distance. This is especially the case in Japanese women who have small physical stature, and this angle can potentially be much larger and therefore caution is necessary.

Lee et al. [16] reported that rotational femoral and tibial articulation mismatch can lead to patellofemoral dysfunction, knee instability, and tibial polyethylene insert wear. Internal rotation of the femoral component moves the groove portion of the femoral component relatively medially, and increases the lateral force vector on the patella [17]. External malrotation of the femoral components could theoretically worsen tibiofemoral congruity and increase the risk of polyethylene insert wear over the long-term, causing residual pain after TKA. Tsukiyama et al. [18] showed that knees with postoperative medial joint laxity $>3^{\circ}$ in flexion resulted in inferior patient satisfaction and knee function as evaluated by the 2011 Knee Society Knee Scoring System. Luyckx et al. [19] reported that posterior translation of the lateral condyle and relative internal tibial rotation during flexion forced stretching or friction of the iliotibial band and caused lateral knee pain in 77 cases $(7.2 \%)$ in a series of 1070 TKAs.

Our study has some limitations. First is the fact that cases are limited to Japanese patients. Because posterior intercondylar distance is predicted to be larger in Western patients than in Asian patients, the impact of cartilage on rotation may likely be smaller on average. Second, all had varus knees and no valgus knees were included, because osteoarthritic valgus knee is rare in Japan. Third, the accuracy of the measurements of the distance between the medial and lateral posterior condyle is possibly not high.

\section{Conclusions}

In cases where the FC external rotation angle is determined using the posterior condyles as landmarks, this

Table 5 Comparison of external rotation angle between subgroups of men divided according to median value

\begin{tabular}{|c|c|c|c|c|c|}
\hline & \multicolumn{4}{|c|}{ Posterior intercondylar distance (subgrouping according to the median value) } & \multirow[t]{3}{*}{$p$-value } \\
\hline & \multicolumn{2}{|c|}{ Short distance group $(\leq 51 \mathrm{~mm})$} & \multicolumn{2}{|c|}{ Long distance group (>51 mm) } & \\
\hline & $\mathrm{n}$ & Data & $\mathrm{n}$ & Data & \\
\hline Angle & 11 & $0.991 \pm 0.783$ & 8 & $1.457 \pm 0.668$ & 0.182 \\
\hline
\end{tabular}

Data are presented as the mean \pm standard deviation. p-value: Welch's t-test 
Table 6 Comparison of external rotation angle between subgroups of women divided according to median value

\begin{tabular}{|c|c|c|c|c|c|}
\hline & & istance (subgro & $\operatorname{ian} a^{2}$ & & $p$-value \\
\hline & & $44 \mathrm{~mm})$ & Lor & $4 \mathrm{~mm})$ & \\
\hline & $\mathrm{n}$ & Data & $n$ & Data & \\
\hline Angle & 20 & $1.800 \pm 0.949$ & 11 & $1.243 \pm 0.628$ & 0.060 \\
\hline
\end{tabular}

Data are presented as the mean \pm standard deviation. $p$-value: Welch's t-test

angle can be affected by the intercondylar distance. This is especially the case in Japanese women who have small physical stature, and this angle can potentially be much larger, and therefore caution is necessary. Our results seem to indicate that several anatomical landmarks should be referenced in order to achieve accurate rotation of the FC.

\section{Abbreviations}

BMl: Body mass index; CT: Computed tomography; FC: Femoral component; HKA angle: Hip-knee-ankle angle; MPR: Multiplanar reformation; MRI: Magnetic resonance imaging; OA: Osteoarthritis; PCA: Posterior condylar axis; PSI: Patient Specific Instruments; TKA: Total knee arthroplasty

\section{Acknowledgements}

Not applicable.

\section{Authors' contributions}

T.M., R.I., and D.K. designed the study. T.M. and S.Y. performed the measurements and analyzed the data. T.M. wrote the manuscript in consultation with M.S., R.I., D.K., and K.M. All authors contributed to the final version of the manuscript. The author(s) read and approved the final manuscript.

\section{Funding}

This research received no specific grant from any funding agency in the public, commercial, or not-for-profit sectors.

\section{Availability of data and materials}

The datasets used and/or analyzed during the current study are available from the corresponding author on reasonable request.

\section{Ethics approval and consent to participate}

All procedures performed in studies involving human participants were in accordance with the ethical standards of the Ethics Committee of Toshima Hospital, Tokyo Metropolitan Health and Hospitals Corporation (approval no. 3161), and with the 1964 Helsinki declaration and its later amendments or comparable ethical standards. Verbal informed consent to participate in the study was obtained at the time of obtaining the surgical consent form, which was taken as documentation of consent to participate in this research. Verbal consent was approved by the ethics committee because this study used only data routinely collected during surgery. In addition, notices are posted on our hospital's website and bulletin boards explaining the use of clinical data for research purposes and informing patients of their right to opt out of the use of their data.

\section{Consent for publication}

Not applicable.

\section{Competing interests}

Dr. Miyasaka reports grants from Japan Medical Dynamic Marketing, Inc., outside the submitted work; Dr. Marumo reports grants from Zimmer Biomet Japan, Inc., grants from Chugai Pharmaceutical Co, LTD., grants from Astellas Pharma Inc., and grants from Asahi Kasei Pharma Corp., outside the submitted work; all other authors declare no conflicts of interest.

Received: 25 March 2020 Accepted: 23 July 2020

Published online: 28 July 2020

\section{References}

1. Merkow RL, Soudry M, Insall JN. Patellar dislocation following total knee replacement. J Bone Joint Surg Am. 1985;67(9):1321-7.
2. Abadie P, Galaud B, Michaut M, Fallet L, Boisrenoult P, Beaufils P. Distal femur rotational alignment and patellar subluxation: a CT scan in vivo assessment. Orthop Traumatol Surg Res. 2009;95:267-71.

3. Berger RA, Crossett LS, Jacobs JJ, Rubash HE. Malrotation causing patellofemoral complications after total knee arthroplasty. Clin Orthop Relat Res. 1998:144-53.

4. Akagi M, Matsusue $Y$, Mata T, Asada Y, Horiguchi M, lida H, et al. Effect of rotational alignment on patellar tracking in total knee arthroplasty. Clin Orthop Relat Res. 1999:155-63.

5. Bäthis H, Perlick L, Tingart M, Lüring C, Zurakowski D, Grifka J. Alignment in total knee arthroplasty. A comparison of computer-assisted surgery with the conventional technique. J Bone Joint Surg Br. 2004;86(5):682-7.

6. Mason JB, Fehring TK, Estok R, Banel D, Fahrbach K. Meta-analysis of alignment outcomes in computer-assisted total knee arthroplasty surgery. J Arthroplast. 2007;22:1097-106.

7. Miyasaka T, Kurosaka D, Saito M, Omori T, Ikeda R, Marumo K. Accuracy of computed tomography-based navigation-assisted total knee arthroplasty: outlier analysis. J Arthroplast. 2017;32:47-52. https://doi.org/10.1016/j.arth.2016.05.069.

8. Nam D, Weeks KD, Reinhardt KR, Nawabi DH, Cross MB, Mayman DJ. Accelerometer-based, portable navigation vs imageless, large-console computer-assisted navigation in total knee arthroplasty. A comparison of radiographic results. J Arthroplast. 2013;28(2):255-61.

9. Jiang J, Kang X, Lin Q, Teng Y, An L, Ma J, et al. Accuracy of patient-specific instrumentation compared with conventional instrumentation in total knee arthroplasty. Orthopedics. 2015;39(Suppl 3):83-7.

10. Asada S, Akagi M, Matsushita T, Hashimoto K, Mori S, Hamanishi C. Effects of cartilage remnants of the posterior femoral condyles on femoral component rotation in varus knee osteoarthritis. Knee. 2012;19:185-9. https://doi.org/10.1016/j.knee.2011.02.008.

11. Fujii T, Kondo M, Tomari K, Kadoya Y, Tanaka Y. Posterior condylar cartilage may distort rotational alignment of the femoral component based on posterior condylar axis in total knee arthroplasty. Surg Radiol Anat. 2012;34:633-8.

12. Hamada D, Wada K, Mikami H, Toki S, Goto T, Tsutsui T, et al. The posterior condylar cartilage affects rotational alignment of the femoral component in varus knee osteoarthritis. J Med Invest. 2017;64(1.2):24-9.

13. Tashiro Y, Uemura M, Matsuda S, Okazaki K, Kawahara S, Hashizume M, et al. Articular cartilage of the posterior condyle can affect rotational alignment in total knee arthroplasty. Knee Surg Sports Traumatol Arthrosc. 2012;20:1463-9.

14. Hanna FS, Teichtahl AJ, Wluka AE, Wang Y, Urquhart DM, English DR, et al. Women have increased rates of cartilage loss and progression of cartilage defects at the knee than men. Menopause. 2009;16(4):666-70.

15. Ishimaru M, Hino K, Onishi Y, Iseki Y, Mashima N, Miura H. A threedimensional computed tomography study of distal femoral morphology in Japanese patients: gender differences and component fit. Knee. 2014;21: 1221-4. https://doi.org/10.1016/j.knee.2014.09.007.

16. Lee DH, Seo JG, Moon YW. Synchronisation of tibial rotational alignment with femoral component in total knee arthroplasty. Int Orthop. 2008;32(2):223-7.

17. Poilvache PL. The patella in total knee replacement: technical aspects on the femoral side. Knee Surg Sports Traumatol Arthrosc. 2001;9(Suppl 1):13-8.

18. Tsukiyama H, Kuriyama S, Kobayashi M, Nakamura S, Furu M, Ito H, et al. Medial rather than lateral knee instability correlates with inferior patient satisfaction and knee function after total knee arthroplasty. Knee. 2017;24(6):1478-84.

19. Luyckx L, Luyckx T, Bellemans J, Victor J. lliotibial band traction syndrome in guided motion TKA. A new clinical entity after TKA. Acta Orthop Belg. 2010; 76(4):507-12.

\section{Publisher's Note}

Springer Nature remains neutral with regard to jurisdictional claims in published maps and institutional affiliations. 